\title{
Correlação socioeconômica e antropométrica em idosos praticantes e não praticantes de exercícios físicos
}

Rev Bras Ativ Fis Saúde p. 121-131

DOI: http://dx.doi.org/10.12820/2317-

1634.2013v18n1p121

1 PUCRS, Porto Alegre, RS-Brasil

2 PAFOR, Cidade, AM, Brasil

\section{Correlation socioeconomic and anthropometric in elderly practitioners and not practitioners of physical activity}

\author{
Janaisa Gomes Dias de Oliveira ${ }^{1}$ \\ Claus Dieter Stobaus ${ }^{1}$ \\ Yukio Moriguchi ${ }^{1}$ \\ Willian Rafael Malezan² \\ Adriano Detoni Filho ${ }^{3}$ \\ Mariana Nolde Pacheco ${ }^{3}$ \\ Larissa Casellani ${ }^{1}$
}

\section{Resumo}

Este estudo, baseado na Tese do Programa de Doutorado em Gerontologia Biomédica, avaliou, a partir de uma análise parcial quantitativa, a correlação do perfil socioeconômico e antropométrico em idosos praticantes e não praticantes de exercícios físicos regulares, assistidos pelo Centro Universitário Vila Fátima e pela Faculdade de Educação Física da PUCRS. Foi selecionada uma amostra inicial de conveniência de 50 idosos, sendo 25 em cada grupo - praticante e não praticante. Do total, observou-se que a maioria era do sexo feminino, com idade entre 62 e 86 anos, ensino fundamental incompleto, cuja renda era acima do salário mínimo e com histórico familiar de doenças cardiovasculares. Entre os grupos ocorreram diferenças significativas nas seguintes variáveis: fumo $(p<0,001)$, hereditariedade $(p<0,001)$, Dobra Cutânea Subescapular (DCS) $(p<0,001)$ e Dobra Cutânea Tricipital(DCT) $(p<0,001)$. Observou-se mudança no percentual de gordura ( $p=0,0001)$, assim como variação de Massa Magra em quilos $(p=0,0293)$ e de massa gorda em quilos entre praticantes e não praticantes $(p=0,0004)$, além da diferença na relação cintura/quadril nesses grupos $(\mathrm{p}=0,0257)$. Nesse sentido, evidencia-se que a prática regular de exercícios físicos em idosos é essencial para a prevenção de doenças, manutenção e recuperação da saúde.

\section{Palavras-chave}

Idoso, Exercício, Saúde.

\begin{abstract}
This study is part of a PhD thesis in Biomedical Gerontology, with partial quantitative analysis, which aimed to correlate on anthropometric and socioeconomic profile of elderly practitioners and non-practitioners of regular exercise, attended at Villa Fatima University Center and the Faculty of Physical Education PUCRS, selecting a convenience sample (initial partial) of 50 seniors (25 practitioners and 25 non-practicing). Of these, we found that the majority were women, between 62 to 86 years, with Incomplete Elementary School, and earned more than minimum wage and with a family history of cardiovascular disease. Between groups (practitioners and non-practitioners) significant differences in the following variables: smoking $(p<0.001)$, heredity $(p<0.001)$, subscapular skinfold (DCS) ( $p$ $<0.001)$ and triceps skinfold (TSF) $(p<0.001)$. Observed difference in fat percentage between the groups ( $p=0.0001)$, as well as the difference of Lean Body Mass in kilograms ( $p=0.0293)$ and fat mass in kilograms between practitioners and non-practitioners $(p=0.0004)$, besides the difference in the waist / hip ratio in these groups $(p=0.0257)$ Therefore, we recommend the regular practice of physical exercise in the elderly, because it is essential for the prevention of disease and the maintenance and restoration of health.
\end{abstract}

\section{Keywords}

Elderly, Exercise, Health. 


\section{INTRODUÇÃO}

Ao longo da vida, o ser humano desenvolve uma série de modificações genéticobiológicas e psicossociais. Uma boa saúde na idade avançada, a qual é entendida como o conjunto de ações realizadas pelo indivíduo diariamente como a sua alimentação, o uso de medicamentos e a prática de atividades físicas ${ }^{1,2}$, depende do estilo de vida adotado desde os primeiro anos.

O envelhecimento significa a fase da vida que começa com a concepção e termina com a morte, descrito como um processo dinâmico, progressivo, irreversível e universal, caracterizado por uma série de modificações morfológicas, fisiológicas, bioquímicas, enzimáticas e psicológicas, em que ocorre a redução da capacidade de adaptação do indivíduo ao meio ambiente, deixando-o vulnerável e exposto a doenças. O envelhecimento biológico determina o declínio harmônico e orgânico, tornando-se mais acentuado depois dos 70 anos, promovendo mudanças funcionais, psicológicas, e perceptivas quanto a autoimagem e autoestima ${ }^{3,4,5 \mathrm{e} 6}$. Estas alterações poderiam ser compensadas pela experiência, consciência, pelo conhecimento e autodomínio dos idosos, contrabalançando as debilidades funcionais.

Os benefícios biopsicossociais obtidos com a prática de ativdade física, incluem mudanças de comportamentos individuais e/ou coletivos, sendo influenciadores da adesão/manutenção de atividades. Essa prática promove saúde e diminui diversos fatores de risco para doenças cardiovasculares ${ }^{7,8}$.

Os idosos passam por transformações em nível psicológico e nutricional as quais se refletem por meio de relações sociais, alterações morfológicas e metabólicas importantes de serem pesquisadas ${ }^{9}$. A antropometria propicia a avaliação do estado nutricional das pessoas, logo, é um método respaldado na área clínica epidemiológica, tanto pela facilidade de manuseio, como por ser de baixo custo, e não invasivo. A maioria dos estudos realizados com populações idosas se utilizou da antropometria e as variáveis mais investigadas foram a massa corporal, a estatura e perímetros corporais.

Conhecer as características demográficas e socioeconômicas, concomitante às informações antropométricas, torna-se imprescindível para que seja possível realizar mapeamento das necessidades da população e orientar políticas de cunho social ${ }^{9}$.

Ressalta-se que o artigo objetivou correlacionar dados entre grupo de idosos praticantes de atividade e não praticante concomitantemente, distinto de outros estudos que apenas destacam resultados em somente um desses grupos.

\section{MÉTOdOS}

Este trabalho caracteriza-se como um estudo observacional do tipo transversal com abordagem descritivo-analítica, assinalado por uma amostra com 50 idosos escolhidos por conveniência.

A pesquisa foi realizada no município de Porto Alegre região ..sul. do Brasil. Os dados foram coletados durante o período de janeiro e dezembro de 2011.

Os idosos foram selecionados a partir das fichas de registro dos atendimentos efetuados pelo Centro Universitário Vila Fátima da PUCRS, de acordo com os critérios de inclusão: assiduidade, faixa etária superior a 60 anos, apto, através de comprovação médica, para a prática de atividades físicas. Foram formados dois grupos distintos: o $1^{\mathrm{o}}$ grupo, com 25 idosos, denominado Praticantes de Atividades Físicas Regulares e o $2^{\circ}$, com 25 idosos, denominado Não Praticantes de Atividades Físicas Regulares. Contrapondo seu perfil sócio-econômico-demográfico 
e seus dados antropométricos, foram analisadas as seguintes variáveis socioeconômicas: faixa etária, renda mensal, escolaridade, moradia, profissão; antropométricas e de saúde: presença de doenças, tabagismo; contrastadas com os perímetros da cintura, quadril, circunferência braquial e da panturrilha, além das dobras tricipital e subescapular. Considerou-se como praticante, o grupo de idosos que participava do programa, regularmente, e era assíduo às aulas; já o não praticante não participava do programa de exercícios físicos.

Todos os participantes realizaram uma entrevista estruturada e uma avaliação física. Foi utilizado um estadiômetro (Sanny Medical), altura de alumínio com precisão de $1 \mathrm{~mm}$, objetivando obter a medida da altura. Os idosos foram medidos descalços, em posição ortostática, com as costas e a parte posterior dos joelhos encostados ao estadiômetro. O peso foi aferido com a balança digital (WELMY/ Classe III), e os mesmos foram pesados apenas com sua roupa íntima. As circunferências da panturrilha $(\mathrm{CP})$ e braquial $(\mathrm{CB})$ foram tomadas na posição bípede, ereto, com os pés separados aproximadamente $20 \mathrm{~cm}$, de modo que o peso fosse distribuído de forma uniforme e a circunferência braquial medida com fita não distensível de metal flexível (Sanny Medical), graduada em centímetros.

A medida das dobras cutâneas subescapular (DCS) e tricipital (DCT) foi mensurada com plicômetro (Cescorf-Mitutoyo KPJ627); na DSC foi mensurada no sentido diagonal - 45 graus de inclinação em relação ao plano horizontal natural e o pinçamento foi exatamente abaixo do ângulo inferior da escápula; enquanto que a DCT foi mensurada no ponto meso-umeral na região posterior do braço ${ }^{10}$.

$\mathrm{Na}$ população idosa, em uma avaliação nutricional utiliza-se mais as seguintes variáveis: peso, estatura, dobras cutâneas e circunferências do braço, da cintura, do quadril e panturrilha. Nessa faixa etária, as alterações referem-se à massa magra e aos padrões de distribuição de gordura, estes sendo frequentes indicadores de riscos cardiovasculares. Os valores de dobra cutânea triciptal (DCT) e dobra subescapular (DCB) correspondem a forma indireta de mensuração da adiposidade corporal, de fácil localização e que apresenta forte correlação com o percentual total de gordura corporal. Estas medidas tendem a reduzir com o avançar do grupo etário, em geral, as mulheres apresentam maior reserva de gordura que os homens. Medidas antropométricas vêm sendo utilizadas tanto para avaliação nutricional de idosos, como para estabelecer níveis de composição corporal, entretanto, a prática isolada desses parâmetros parece ter baixa especificidade, em função da heterogeneidade de fatores típicos dessa faixa etária ${ }^{11}$.

Para a análise dos dados foi utilizado o software GraphPad Prism 5.0 e programa estatístico Statistical Package for Social Sciences versão 17.0 (SPSS Inc., Chicago, IL, USA, 2008), sendo que para critérios de decisão estatística adotou-se o nível de significância de 5\%. Na comparação das variáveis categóricas entre os dois grupos foi utilizado o teste Qui-quadrado de Pearson, e nas situações em que não foram satisfeitos os pressupostos básicos foi utilizado o teste Exato de Fisher. A análise que envolveu a comparação das variáveis quantitativas foi utilizado o teste de Mann Whitney.

As atividades físicas eram conduzidas pelos alunos de Educação Física, supervisionados pelo seu professor, na disciplina estágio IV- Saúde e lazer, na Faculdade de Educação Física, duas vezes por semana, durante 50 minutos, com intensidade moderada, variando os exercícios (força, flexibilidade, coordenação, equilíbrio) e atividades lúdicas. $\mathrm{O}$ grupo de praticantes de atividades físicas regulares, participava do programa cerca de 4 anos, realizando atividades tais como: caminhadas com ou sem obstáculos, exercícios para membros superiores com bastões ou halteres, 
exercícios com bola, atividades aeróbicas e de relaxamento, entre outras. Sendo que este grupo não realizava atividades externas, somente as atividades ministradas pelos acadêmicos.

\section{RESULTADOS}

A amostra foi composta por 25 idosos praticantes e 25 idosos não praticantes de exercícios físicos, sendo 9 homens (18\%) e 41 mulheres (82\%). No grupo de não praticantes foram observados os seguintes aspectos: maior concentração na faixa etária de 65-74 anos (48\%); escolaridade mais frequente foi o ensino fundamental incompleto (76\%); a maioria tinha casa própria (88\%) e estava aposentada (60\%); a renda familiar mensal concentrava-se entre 1 e 5 salários mínimos (56\%). Quanto à presença de doenças, 96\% confirmaram estar com alguma enfermidade. Um percentual de idosos afirmou não possuir hipertensão arterial sistêmica (76\%) e dislipidemia (24\%). Alguns idosos apresentaram outras patologias associadas, como diabetes (32\%) e obesidade (12\%), e a grande maioria (96\%), apresentou fatores hereditários para suas doenças atuais.

No grupo dos idosos praticantes de atividades físicas regulares, observou-se um percentual de faixa etária de 60-64 anos (40\%), havendo predomínio do sexo feminino (96\%), com ensino fundamental incompleto (48\%), sendo (12\%) analfabetos. Os idosos desse grupo moravam em casa própria, sendo a maioria aposentados (80\%); com renda familiar mensal entre 1 e 5 salários mínimos (60\%). Quanto à presença de doenças, (96\%) apresentavam algum tipo de patologia isolada, enquanto $60 \%$ tinham outras doenças associadas. Destes idosos praticantes, $80 \%$ apresentaram fator de hereditariedade para as doenças declaradas, 56\% não apresentavam hipertensão arterial, nem dislipidemia (40\%) e 16\% eram fumantes. Existe associação entre os grupos (não praticantes e praticantes) em relação ao gênero $(p=0,001)$.

$\mathrm{Na}$ análise econômica, foi constatado que $94 \%$ dos idosos estudados possuem casa própria, $58 \%$ obtêm uma renda entre 1 e 5 salários mínimos e $80 \%$ encontram-se aposentados.

Avaliando as variáveis que apontaram associação ou diferenças significativas entre os dois grupos, verificou-se que, a média de idade dos praticantes $(65,85 \pm$ $5,94)$ mostrou-se significativamente $(\mathrm{p} \leq 0,001)$ menor que a dos não praticantes $(72,15 \pm 6,75)$.

Em relação a escolaridade, a associação significativa $(\mathrm{p} \leq 0,001)$ apontou que o grupo dos praticantes mostrou-se relacionado aos níveis de escolaridade mais elevadados, enquanto que, com o grupo dos não praticantes o nível de estudo estava associado com o ensino fundamental incompleto.

Acerca do rendimento médio, observou-se que o grupo de praticantes de atividade física mostrou-se associado significativamente as faixas de renda de valores mais elevados, enquanto que, com o grupo de não praticantes a associação se configurou nas faixa de rendimento de menor valor $(\mathrm{p}=0,026)$.

Quanto à presença de co-enfermidades, a diferença significativa também se configurou ( $\mathrm{p}<0,0001)$, de forma que, os praticantes apresentaram mais patologias concomitantes além das citadas, quando comparados ao grupo de não praticantes.

Em relação às variáveis de presença de outro tipo de patologia e escolaridade, estas também foram estatisticamente significativas, assim como, entre as variáveis diabetes e escolaridade $(\mathrm{p}=0,001)$ e, entre a hereditariedade e idade das pessoas investigadas $(\mathrm{p}=0,005)$. 
Sobre a diferenças entre os grupos em relação as variáveis antropométricas (Figura 1), observou-se que na DCT a média dos praticantes $(22,74 \pm 7,54 \mathrm{mi}-$ límetros) foi mais elevada ( $\mathrm{p}<0,001)$ que nos não praticantes $(15,93 \pm 5,15$ milímetros). Para a DSC a diferença na média no grupo dos praticantes $(20,01 \pm 5,74$ milímetros) e dos não praticantes $(13,63 \pm 3,96$ milímetros $)$ mostrou-se expressiva $(\mathrm{p}<0,001)$.

$\mathrm{Na}$ figura 2, a diferença de percentual de gordura entre os praticantes $(29,36 \pm$ $5,5) \%)$ e não praticantes $(21,68 \pm 4,5 \%$. $)$ mostrou-se estatisticamente significativa $(\mathrm{p}<0,001)$.

Ainda verificou-se que a diferença de peso entre os grupos praticantes $(68,16$ $\pm 10,04 \mathrm{~kg})$ e não praticantes $(66,44 \pm 9,68)$ não foi significativa $(\mathrm{p}=0,5917)$. Para o IMC a diferença significativa também não se configurou apontando que as variações entre os grupos praticantes $(28,15 \pm 3,62 \mathrm{Kg} / \mathrm{m} 2)$ e não praticantes $(27,6$ $\pm 3,73 \mathrm{Kg} / \mathrm{m} 2)$ não foram relevantes neste estudo ( $\mathrm{p}=0,3918$ ).

Em relação a estatura, as médias mostraram-se estatisticamente semelhantes ( $\mathrm{p}=0,5378)$, entre os grupos dos praticantes $(1,55 \pm 0,08$ metros $)$ e não praticantes $(1,55 \pm 0,08$ metros).

$\mathrm{Na}$ figura 3, a diferença de massa magra entre os grupos de praticantes e não praticantes foi expressiva ( $\mathrm{p}=0,0293)$, com média dos praticantes $(47,76 \pm 4,9$ quilos) inferior a dos não praticantes $(51,93 \pm 7,47$ quilos). E na massa gorda entre o grupo anterior, também mostrou-se significativa ( $\mathrm{p}<0,001)$, com média dos praticantes $(20,4 \pm 6,22$ quilos) superior a dos não praticantes $(14,51 \pm 4,06$ quilos).

Dobra Tricipital

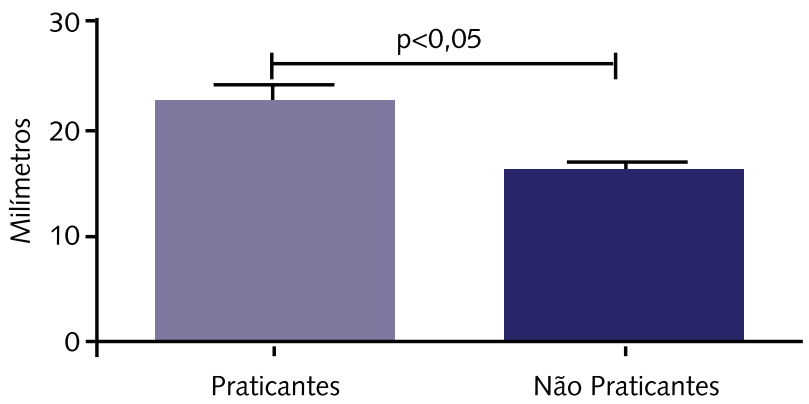

Dobra Subescapular

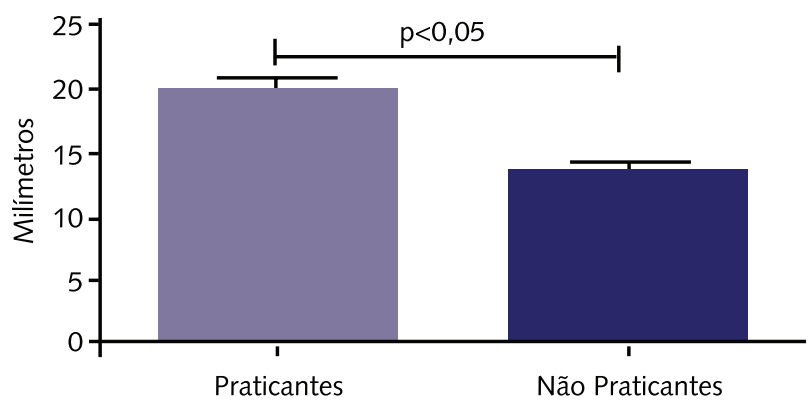

Figura 1 - Valores em percentuais relativos a avaliação de dobras triciptal e subescapular entre grupo de idosos praticantes comparativamente ao grupo de idosos não praticantes de atividades físicas regulares.

Percentiual de Gordura \%

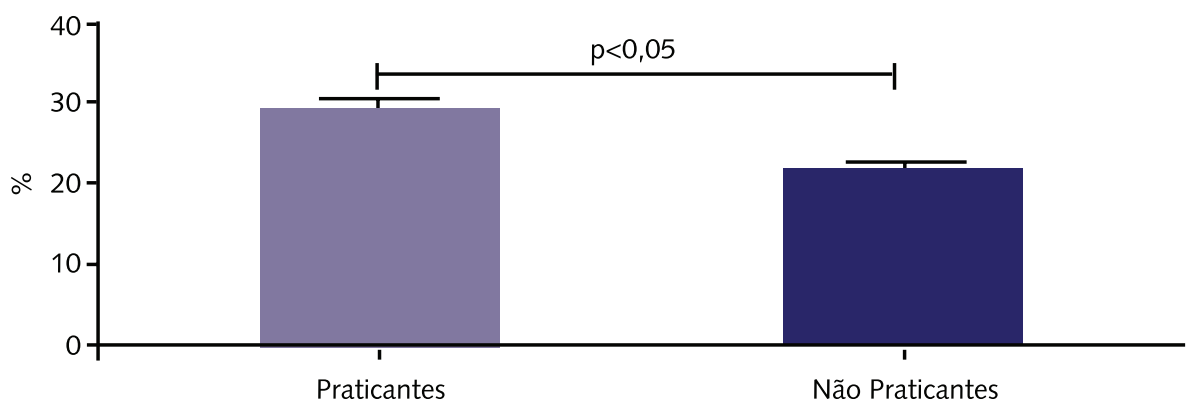

Figura 2 - Valores em percentuais relativos ao percentual de gordura entre grupo de idosos praticantes comparativamente ao grupo de idosos não praticantes de atividades físicas regulares. 

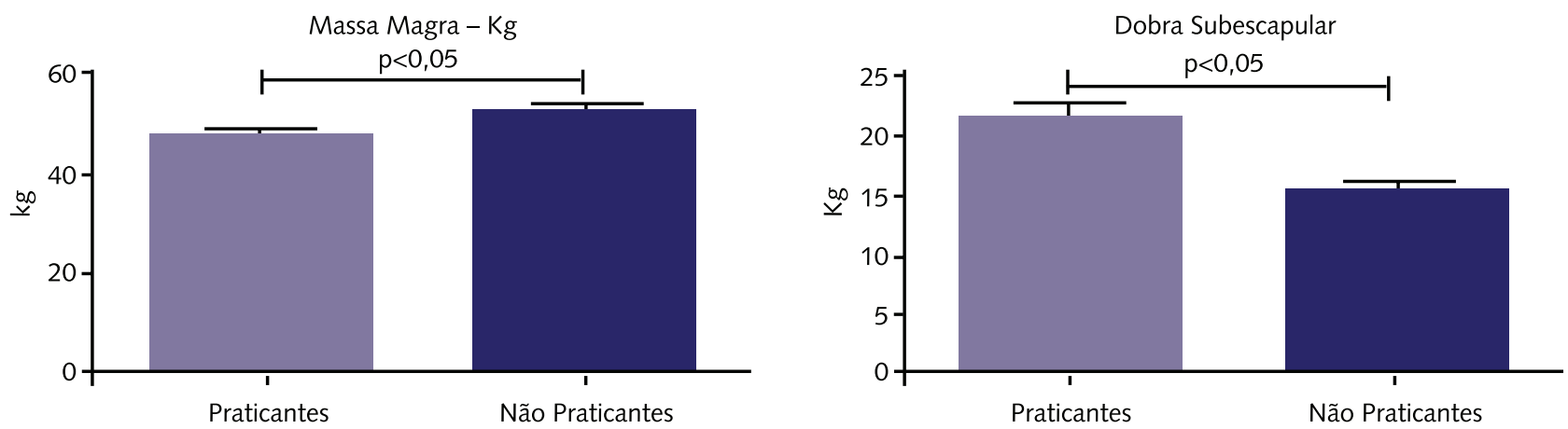

Figura 3 - Valores relativos ao percentual de massa magra e massa gorda entre grupo de idosos praticantes comparativamente ao grupo de idosos não praticantes de atividades físicas regulares.

A diferença da CP entre os grupos foi irrelevante $(\mathrm{p}=0,6964)$, indicando que as diferenças observadas entre as médias dos praticantes $(36,88 \pm 2,67$ centímetros) e não praticantes $(36,71 \pm 2,82$ centímetros) se deve ao acaso.

Estatisticamente, a diferença da CB entre o grupo de praticantes $(30,63 \pm 2,82$ centímetros) e não praticantes $(31,02 \pm 3,23$ centímetros $)(\mathrm{p}=0,9033)$ apresentou-se ínfimo. Bem como a diferença na cirtrometria do quadril entre os grupos estudados ( $p=0,4779)$, embora a média dos praticantes $(102,5 \pm 9,06$ centímetros $)$ tenha se mostrado inferior a dos não praticantes (100,8 $\pm 11,52$ centímetros).

Além disso, o diferencial da cirtometria da cintura entre esses grupos também não foi significativo ( $p=0,2191)$, sendo a média dos praticantes $(94,83 \pm 13,21$ centímetros) e dos não praticantes $(99,2 \pm 11,51$ centímetros) não diferiu de forma relevante.

Já a diferença da relação ICQ foi expressiva $(p=0,0257)$ entre os grupos, uma vez que a média dos praticantes $(0,92 \pm 0,1 \mathrm{C} / \mathrm{Q})$ mostrou-se menor que a dos não praticantes $(0,98 \pm 0,04 \mathrm{C} / \mathrm{Q})$. Pode-se dizer que estas medidas são significativas entre os grupos pesquisados, pois relacionam-se com depósito de gordura.

As equações mais utilizadas para cálculo de percentual de gordura envolvem o uso de diversas dobras corporais. Ocorre que, em função das modificações fisiológicas do idoso, muitas dessas medidas de dobras não se aplicam. A mais recomendada para o idoso, com menor erro é a dobra tricipital, seguida pela subescapular.

No estudo, foi encontrada diferença estatisticamente relevante $(p \leq 0,001)$ entre sobrepeso e obesidade em idosos, principalmente nos idosos não praticantes.

\section{DISCUSSÃO}

Os participantes desta pesquisa foram, na maioria, do sexo feminino - homens (18\%) e mulheres (82\%). Analisando a associação entre os grupos de não praticantes e praticantes, quanto ao gênero, depreendeu-se que mulheres têm estilo de vida mais ativo que homens, provavelmente em função de suas atividades domésticas ${ }^{12,13}$.

Destaca-se que a participação feminina na sociedade vem aumentando nesses últimos anos, sugerindo que a baixa escolaridade apresentada relaciona-se com as idosas que não puderam estudar em sua época e seu contexto ${ }^{14}$.

Ainda com relação a escolaridade, a associação significativa ( $\mathrm{p} \leq 0,001)$, apontou que o grupo dos praticantes de atividades físicas apresentou níveis de escolaridade elevados, sendo que o grupo dos não praticantes prevaleceu o ensino fundamental incompleto. $\mathrm{O}$ grupo de praticantes demonstrou renda mais elevadas, enquanto que, o grupo de não praticantes apresentou faixa de rendimento de menor valor $(\mathrm{p}=0,026)$.

A relevância destes dados consiste no fato de que a prática de atividades fisicas regulares requer uma escolaridade mínima para a participação e entendimento 
das atividades propostas pelos acadêmicos. Os dados deste estudo demonstraram que os idosos não praticantes do programa supervisionado de atividades físicas, optaram por atividades lúdicas, artesanais, e de convivência, ao invés de exercícios físicos. Apesar das atividades desenvolvidas neste grupo não exigirem níveis escolares elevados, e serem de fácil aprendizagem, eram igualmente valorizadas pelos idosos participantes. O grupo dos praticantes, que apresentou maior renda, parecia compreender a importância de sua participação no programa, a assiduidade, e influência no controle de patologias crônico-degenerativas. Assim, neste estudo contastou-se que os idosos mais escolarizados tiveram maior adesão e valorizaram mais a prática de atividades físicas, pois entendiam como método preventivo e não farmacológico contra o aparecimento de doenças.

Assim, notou-se que idosos mais instruídos intelectualmente cuidavam-se mais, em relação aos demais. A divulgação sobre a melhora da qualidade de vida por meio da prática de atividades físicas é cada vez maior, entretanto, grande parte da população idosa ainda não participa regularmente de atividades físicas. A formação escolar maior reflete mais adesão à atividade física, mostrando que diferenças de escolaridade repercutem nas atitudes, concepções e escolhas das atividades físicas de interesse ${ }^{15}$.

$\mathrm{Na}$ análise econômica, destaca-se que segmentos socioeconômicos menos favoráveis são preditores de baixo nível de atividade física ${ }^{16}$. Além disso, estudos ${ }^{17}$, ${ }^{18}$ destacam que fatores socioeconômicos podem levar a um quadro de isolamento social, podendo comprometer notoriamente a saúde e a qualidade de vida, sabendo-se que pessoas com nível de escolaridade superior têm mais acesso às informações referentes de doenças.

No estudo foi encontrada uma relação entre adesão à atividade física e renda. Parece que grupos com baixa renda podem ter a ociosidade reforçada, uma vez que possuem maior probabilidade de serem relativamente desinformados sobre os benefícios para a saúde advindos da atividade. Além disso, como consequência da vida atual, o idoso muitas vezes tem que arcar com os custos de manutenção de sua casa, sendo o valor da aposentadoria inferior aos vencimentos de seu período produtivo e também sua única fonte de renda. Por outro lado, o evento da aposentadoria proporciona maior tempo livre, e pode influenciar diretamente em maior participação em programas de atividade física ${ }^{19}$.

Os idosos praticantes apresentaram um número maior patologias concomitantes além das citadas, quando comparado aos não praticantes ( $\mathrm{p}<0,0001)$. Estimase que os idosos praticantes da atividades do programa realizavam-as como complemento do tratamento de patologias prévias. Logo, mais uma vez a influência da escolaridade, é apontada no surgimento de possíveis agravamentos patológicos, logo, acredita-se que, neste estudo, os idosos mais escolarizados tinham um cuidado maior. Já em relação a hereditariedade, observou-se que, quanto mais alta faixa etária , maior a chance de riscos cardiovasculares.

Quanto às variáveis - presença de outras patologias e escolaridade, diabetes e escolaridade, hereditariedade e idade, foram estatisticamente significativas, neste estudo. A idade avançada não implica necessariamente no surgimentoto de patologias hereditárias. As doenças hereditárias são genéticas e podem repassadas de geração em geração, manifestando-se em algum momento de suas vidas, dependendo da predisposição genética individual ${ }^{20}$.

Em relação à significância estatística entre hereditariedade e fumo, sugere-se que idosos que foram expostos ao fumo e nicotina no período gestacional podem demonstrar maior dependência via sistema nervoso central à nicotina. $\mathrm{O}$ ato de fumar não é hereditário, mas a dependência neurológica via centros do prazer leva 
à adição futura. Apesar de fatores ambientais estarem envolvidos, estima-se que fatores genéticos possam ter uma responsabilidade superior a $50 \%$ na iniciação e dependência tabágica e, ainda maior, entre 70-86\%, na manutenção do hábito e na quantidade de cigarros fumados por $\operatorname{dia}^{21}$.

Entre as variáveis antropométricas, observou-se que na DCT a média dos praticantes foi mais prevalente $(\mathrm{p}<0,001)$ que nos não praticantes. Para a DSC a diferença na média no grupo dos praticantes e dos não praticantes foi expressiva $(p<0,001)$ As equações mais utilizadas para cálculo de percentual de gordura envolvem o uso de diversas dobras corporais. Ocorre que em função das modificações fisiológicas do idoso, muitas dessas medidas de dobras não se aplicam. A mais recomendada para o idoso, com menor erro é a dobra tricipital, seguida pela subescapular.

Os valores de DCT são congruentes às informações disponíveis na literatura que indicam maior acúmulo de gordura nas extremidades das mulheres, quando comparadas aos homens. Semelhantemente aos presentes resultados, autores encontraram elevação nas medidas de DCT nos homens, constatados em estudos que apresentaram tanto os dados em percentual como o aumento dos valores médios de DCT nos homens até os 79 anos, quando, então, ocorre diminuiçãa ${ }^{22}$. Com isso, é possível observar que independentemente do grupo etário, existe tendência de maiores depósitos de gordura entre as mulheres e que a diminuição de massa gorda torna-se mais pronunciada nos idosos mais velhos.

A diferença de peso entre os grupos praticantes e não praticantes e os valores de IMC não foram significativos. Ambos os grupos apresentaram as médias de estaturas, semelhantes, e sem significância estatística.

A correlação do IMC com a espessura das dobras triciptal e subescapular na população adulta americana variou de 0,61 a 0,76 , mostrando boa correlação do índice com medidas de adiposidade. O perímetro da cintura é indicador da distribuição abdominal da gordura e também da gordura corporal total. As mudanças relacionadas com o acúmulo da gordura visceral ou subcutânea associadas ao processo de envelhecimento podem ser afetadas tanto pela quantidade inicial de tecido adiposo como pelo aumento da massa corporal. Essas transformações ocorrem de forma diferente entre homens e mulheres e características genéticas são fatores predisponentes para a centralização ${ }^{23}$.

A diferença de massa magra entre os grupos de praticantes e não praticantes de atividades físicas regulares foi expressiva $(p=0,0293)$. A massa gorda entre o primeiro grupo, mostrou-se significativa $(\mathrm{p}<0,001)$. Aponta-se que os praticantes apresentaram maior percentual de gordura em relação aos não praticantes. Estima-se que os idosos não praticantes, apesar de não aderirem a programas supervisionados de exercícios físicos regulares, tinham seu gasto calórico contrabalançado pela realização de atividades instrumentais da vida diária, atividades laborais, entre outros. Além disso, estes idosos, participavam de reuniões semanais com diversos profissionais de saúde, nas quais eram abordados diversos assuntos relacionados a prevenção de patologias e agravos mais comuns no processo de envelhecimento. Assim, estes idosos apesar de baixa escolaridade, tinham acesso a informações sobre seu estado de saúde, entre eles, o controle de obesidade e riscos cardiovasculares. Os idosos praticantes, por sua vez, provavelmente já portavam patologias, e estavam acima do peso, antes de ingressarem no programa supervisionado, o que pode explicar a presença de maior percentual de gordura corporal.

Estudos analisaram a distribuição de gordura em mulheres de diferentes grupos etários por meio de tomografia computadorizada, constatando que o envelhe- 
cimento leva a redistribuição e internalização da gordura abdominal, principalmente entre as mulheres ${ }^{24}$.

Existem inúmeros benefícios que o exercício físico orientado pode oferecer aos idosos incluindo o controle ou diminuição da gordura corporal, manutenção ou incremento da massa muscular, aumento de força muscular e densidade óssea, fortalecimento do tecido conectivo e melhora da flexibilidade ${ }^{25}$.

Uma pesquisa ${ }^{26}$ identificou a valorização e o reconhecimento de 40 idosos em relação à importância da prática de atividades físicas sistematizadas e sua influência na autopercepção em relação às atividades de vida diária, demonstrando melhora nos aspectos sociais, psicológicos e na relação interpessoal de idosos praticantes de atividades físicas. Além disso, outro estudo ${ }^{27}$ que avaliou 168 indivíduos entre 60 e 95 anos de idade, também apontou melhores índices de satisfação com a vida, autoestima e crescimento pessoal em idosos ativos, ressaltando a importância de exercícios físicos regulares em idosos.

O diferencial da cirtometria da cintura entre esses grupos não foi significativo $(p=0,2191)$, já a diferença da Relação ICQ foi significativa $(p=0,0257)$ entre os grupos. No estudo, foi encontrada diferença significativa $(p \leq 0,001)$ entre sobrepeso e obesidade em idosos, principalmente nos idosos não praticantes de exercícios físicos regulares. Considerando que em função do efeitos fisiológicos do envelhecimento, o metabolismo do idoso tende a ser mais lento; combinado com alimentação inadequada, rica em açúcares e gorduras, e não contrabalanceada com a prática de atividades físicas aeróbicas; estes fatores propiciam o sobrepeso e consequentemente o aparecimento de doenças coronarianas.

Estudo $^{28}$ avaliou diferenças antropométricas de 28 idosos de regiões brasileiras urbanas e rurais, obtendo como resultado uma taxa elevada de sobrepeso e obesidade em idosos da região urbana se comparados aos idosos da área rural. Este resultado se deve ao fato de que na zona rural, as pessoas relataram trabalhar na lavoura, fazendo suas atividades diárias a pé e consumindo alimentos naturais, diferentemente da cidade onde os indivíduos andam mais de carro, consomem mais alimentos industrializados e não praticam tanta atividade física.

Em relação ao sobrepeso e a obesidade, trabalho ${ }^{29}$ destaca que $50 \%$ dos idosos com sobrepeso, mostrando um número alarmante que resulta na necessidade da criação de alternativas e implantação de oficinas com exercícios físicos e reeducação nutricional.

A adequação do estilo de vida individual e populacional remete à prevenção primária geriátrica e inclui aspectos relacionados à nutrição, atividades físicas, repouso, controle da obesidade e do tabagismo. O controle destes fatores visa diminuir e/ou retardar o aparecimento de disfunções e morbidades crônico-degenerativas associadas ao envelhecimento ${ }^{30}$.

\section{CONCLUSÕES}

O presente estudo apresentou resultados significativos, porém, diferentemente de outros artigos, foram apresentadas informações inovadoras: o fato dos idosos praticantes de exercícios físicos regulares desta amostra, serem mulheres, mais escolarizadas e apresentarem maior percentual de gordura, bem como a presença de mais patologias concomitantes além das questionadas na avaliação, quando comparados ao grupo de não praticantes. Existem registros, na literatura, de comprovações de resultados positivos - benefícios aeróbicos, redução de medidas e prevenção contra doenças sistêmicas - adotando esta frequência semanal e tempo, e 
atividades realizadas semelhantes às adotadas neste programa. Todavia, este estudo sugere a necessidade de incremento de pesquisas na área, na tentativa de melhores esclarecimentos quanto a esta questão em especial, sobre a influência da prática de exercícios físicos, níveis de massa magra e gorda e a presença de patologias.

\section{REFERÊNCIAS}

1. Cardoso JR. Corpo e Envelhecimento. A Terceira Idade, SESC - São Paulo, 2002;13(25):76-85.

2. Brasil. Ministério da Saúde - Organização Mundial da Saúde. Trabalhando juntos pela saúde / Organização Mundial da Saúde. Brasília: Ministério da Saúde, 2007. 210 p.

3. Papaléo-Neto M. O estudo da velhice no século XX: histórico, definição do campo e termos básicos. In: Freitas E, et al., organizadores. Tratado de Geriatria e Gerontologia. Rio de Janeiro: Guanabara Koogan; 2002. p. 2-12.

4. Terra N, Oppermann R, Terra P. Doenças geriátricas \& exercícios físicos. Porto Alegre: EDIPUCRS; 2010.

5. Freitas E, et al., organizadores. Tratado de Geriatria e Gerontologia. Rio de Janeiro: Guanabara Koogan; 2002. p. 13-19.

6. Mazo GZ, Lopes MA, Benedetti TB. Atividade Física e o Idoso: concepção gerontológica, Porto Alegre, RS: Sulina; 2001.

7. Maciel MG. Atividade Física e Funcionalidade do Idoso. Rio Claro. Revista Motriz 2010;16(4):1024-1032.

8. Farinatti PTV. Envelhecimento: promoção da saúde e exercício: bases teóricas e metodológicas. Barueri: Manole; 2008. p. 39-54.

9. SILVA DAS. Perfil Síciodemográfico e Antropométrico. Estud. interdiscipl. envelhec., Porto Alegre, 2011;16(1):23-39.

10. Machado FA. Dobras Cutâneas: Localização e Procedimentos. Revista de Desporto e Saúde, SP. p. 41-45, 2005.

11. Frisanho AR. Anthropometric standards for the assessment of growth and nutritional status. Ann Arbor, University of Michigan Press, 1990.

12. Mazo GZ, Mota J, Gonçalvez LHT, Matos MG. Nível de atividade física, condições de saúde e características sócio-demográficas de mulheres idosas brasileiras. Revista Portuguesa de Ciências do Desporto. Portugal, 2005;5(2):202-212.

13. Malta DC, Moura EC, Castro AM, Cruz DKA, Morais Neto OL, Monteiro CA. Padrão de atividade física em adultos brasileiros: resultados de um inquérito por entrevistas telefônicas, 2006. Epidemiologia Serv Saúde 2009;18(1):7-16.

14. Mosquera JJM. Vida Adulta. 3a . ed. Porto Alegre: Sulina,1983.

15. Duarte PR, Gonçalves AK, Santos CL. A concepção de pessoas de meia-idade sobre saúde, envelhecimento e atividade física como motivação para comportamentos ativos. Rev Bras Ciênc Esporte 2002;23(3):35-48.

16. Zaitune MPA, Barros MBA, César CLG, Carandina L, Goldbaum M. Fatores associados ao sedentarismo no lazer em idosos, Campinas, São Paulo, Brasil Cad. Saúde Pública, Rio de Janeiro 2007;23(6):1329-1338.

17. Mota-Pinto A, Rodrigues V, Botelho A, Veríssimo MT, Morais A, Alves C, et al. A sociodemographic study of aging in the Portuguese population: The EPEPP study. Archives of Gerontology and Geriatrics 2011;52(3):304-308.

18. Ortiz MCA, Zanetti ML. Levantamento dos Fatores de Risco para Diabete Mellitus Tipo 2 em Uma Instituição de Ensino Superior. Rev Latino-am Enfermagem 2001;9(3):58-63.

19. Andreotti MC, Okuma SS. Perfil sócio-demográfico e de adesão inicial de idosos ingressantes em um programa de educação física. Rev Paul Educ Física 2003;17(2):142-53.

20. Pasternak JJ. Genética Molecular Humana. Mecanismos de Doenças hereditárias. $1^{\mathrm{a}}$ ed. Manole; 2002. p. 365-412.

21. Santos V. Inter-relações entre tabagismo, sintomas depressivos e genética/Porto Alegre: PUCRS, 2011.

22. Menezes TN, Marucci MFN. Antropometria de idosos residentes em instituições geriátricas, Fortaleza, CE. Rev Saúde Pública 2005;39(2):169-75. 
23. Santos DM, Sichieri R. Índice de massa corporal e indicadores antropométricos de adiposidade em idosos. Rev Saúde Pública. SP, 2005;39(2):163-8.

24. Zamboni M, Turcato E, Santana H, Maggi S, Harris TB, Pietrobelli A, et al. The relationship between body composition and physical performance in older women. J Am Geriatr Soc 1999;47(12):1403-1408.

25. Nelson ME, Rejeski WJ, Blair SN. Physical activity and public health in older adults: recommendation from the American College of Sports Medicine and the American Heart Association. Circulation 2007;166(9):1094-105.

26. Kuwano VG, Silveira AM. A Influência da Atividade Física Sistematizada na Autopercepção do Idoso em Relação às Atividades da Vida Diária. Revista da Educação Física - UEM, 2002;13(2):35-40.

27. Fernandes HM, Raposo JV, Pereira E, Ramalho J, Oliveira S. A influência da atividade física na saúde mental positiva de idosos. Motricidade 2009;5(1):33-50.

28. Tavares EL, Anjos LA. Perfil antropométrico da população idosa brasileira. Resultados da pesquisa nacional sobre saúde e nutrição. Cad. Saúde Pública 1999;15(4):759-68.

29. Santos DM, Sichieri R. Índice de massa corporal e indicadores antropométricos de adiposidade em idosos. Rev Saúde Pública 2008;39(2):63-8.

30. Freitas E, et al., organizadores. Tratado de Geriatria e Gerontologia. Rio de Janeiro: Guanabara Koogan; 2002. p. 603-608.

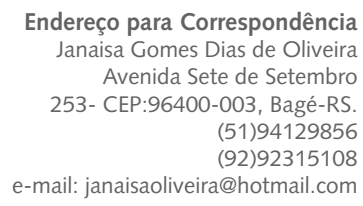

